\title{
Objective Functions with Redundant Domains
}

\author{
Fatima Affif Chaouche, Carrie Rutherford ${ }^{\dagger}$ Robin Whitty ${ }^{\dagger}$
}

February 21, 2012

\begin{abstract}
Let $(E, \mathcal{A})$ be a set system consisting of a finite collection $\mathcal{A}$ of subsets of a ground set $E$, and suppose that we have a function $\phi$ which maps $\mathcal{A}$ into some set $S$. Now removing a subset $K$ from $E$ gives a restriction $\mathcal{A}(\bar{K})$ to those sets of $\mathcal{A}$ disjoint from $K$, and we have a corresponding restriction $\left.\phi\right|_{\mathcal{A}(\bar{K})}$ of our function $\phi$. If the removal of $K$ does not affect the image set of $\phi$, that is $\operatorname{Im}\left(\left.\phi\right|_{\mathcal{A}(\bar{X})}\right)=\operatorname{Im}(\phi)$, then we will say that $K$ is a kernel set of $\mathcal{A}$ with respect to $\phi$. Such sets are potentially useful in optimisation problems defined in terms of $\phi$. We will call the set of all subsets of $E$ that are kernel sets with respect to $\phi$ a kernel system and denote it by $\operatorname{Ker}_{\phi}(\mathcal{A})$. Motivated by the optimisation theme, we ask which kernel systems are matroids. For instance, if $\mathcal{A}$ is the collection of forests in a graph $G$ with coloured edges and $\phi$ counts how many edges of each colour occurs in a forest then $\operatorname{Ker}_{\phi}(\mathcal{A})$ is isomorphic to the disjoint sum of the cocycle matroids of the differently coloured subgraphs; on the other hand, if $\mathcal{A}$ is the power set of a set of positive integers, and $\phi$ is the function which takes the values 1 and 0 on subsets according to whether they are sum-free or not, then we show that $\operatorname{Ker}_{\phi}(\mathcal{A})$ is essentially never a matroid.
\end{abstract}

Keywords: matroid, optimization, objective function, duality, Turán-type problems.

\section{Introduction}

We are motivated by a very general decision problem in combinatorial optimisation:

Let $(E, \mathcal{A})$ be a set system consisting of a finite collection $\mathcal{A}$ of subsets of a ground set $E$. Let $\phi$ be a mapping from $\mathcal{A}$ to some set $S$ and let $S^{\prime}$ be a subset of $S$. Is there some set $X$ in $\mathcal{A}$ for which $\phi(X) \in S^{\prime}$ ?

The greedy algorithm answers this question when: (1) $E$ is a weighted set over, say, $\mathbb{R}$; (2) $\phi$ maps each set in $\mathcal{A}$ to the sum of the weights of its elements; (3) $S^{\prime}=[0, k]$, for some $k \in \mathbb{R}$; and, crucially, (4) $\mathcal{A}$ is the collection of independent sets of a matroid on ground set $E$.

\footnotetext{
*University of Sciences and Technology Houari Boumediene, Algiers

${ }^{\dagger}$ London South Bank University
} 
Example 1 Suppose $G=(V, E)$ is a graph with vertex set $V$ and edge set $E$, and $\mathcal{A}$ is the collection of forests in $G$. Suppose the edges are weighted with weight function $w: E \rightarrow \mathbb{R}^{\geq 0}$ and, for $X \subseteq E$, let $w(X)$ denote the sum of the edge weights in $X$, i.e. $w(X)=\sum_{e \in X} w(e)$. Define two functions from $\mathcal{A}$ to $\mathbb{R}^{\geq 0}$ :

$$
\begin{aligned}
\phi_{1}: F & \mapsto\left\{\begin{array}{cl}
w(F) & F \text { is a spanning tree } \\
0 & \text { otherwise; }
\end{array}\right. \\
\phi_{2}: F & \mapsto\left\{\begin{array}{cl}
w(F) & F \text { is a path } \\
0 & \text { otherwise. }
\end{array}\right.
\end{aligned}
$$

for $F \in \mathcal{A}$. Now the question "Does $\mathcal{A}$ contain a set $X$ with $\phi_{1}(X) \in[0, k]$ ?" can be solved by the greedy algorithm; but the question "Does $\mathcal{A}$ contain a set $X$ with $\phi_{2}(X) \in[k, \infty)$ ?" is the problem of finding a maximum-weight path in $G$ which is NP-hard.

A strategy for the general decision problem might be to look for a subset $K \subseteq E$ such that the image of $\phi$ is unchanged when the ground set is restricted to $E-K$, that is

$$
\operatorname{Im}\left(\left.\phi\right|_{\mathcal{J}}\right)=\operatorname{Im}(\phi), \text { where } \mathcal{J}=\{X \in \mathcal{A} \mid X \cap K=\emptyset\} .
$$

This does not solve the decision problem directly but instead tries to reduce it to a 'smaller' problem.

We shall say that a subset $K$ of $E$ which satisfies condition (1) is a kernel set, in analogy with the usual notion of the kernel of a function: $K$ is a part of the domain which can be regarded as contributing trivially to the image of $\phi$.

We are now faced with the alternative problem of reducing the domain over which we must optimise: given $\phi$, maximise the cardinality of $K \subseteq E$ over the collection of all subsets $K$ which are kernel sets for $\phi$. If this collection happens to be the collection of independent sets of a matroid then this reduction problem, at least, can be solved greedily.

The greedy algorithm supposes the existence of an oracle to answer the question "Is set $X$ independent?" This question may itself be hard to answer; however, our concern here is simply with the question:

Does the set system $(E,\{K \subseteq E \mid K$ satisfies equation (1) $\})$ form a matroid (defined in terms of its independent sets) on $E$ ?

We shall refer to the set system in question (2) as the kernel system for $(E, \mathcal{A})$ with respect to $\phi$ and denote it by $\operatorname{Ker}_{\phi}((E, \mathcal{A}))$, or by $\operatorname{Ker}_{\phi}(\mathcal{A})$ or simply $\operatorname{Ker}_{\phi}$ where this causes no ambiguity ${ }^{1}$.

Notice that the set system constituting $\operatorname{Ker}_{\phi}((E, \mathcal{A}))$ is certainly closed under taking subsets. If it satisfies the independence axioms of a matroid over $E$ we call it the kernel matroid of $(E, \mathcal{A})$ with

\footnotetext{
${ }^{1}$ This definition bears no relation, as far as we know, to kernel systems as defined for directed graphs by Frank [4]. Our adoption of the term should, we hope, cause no confusion.
} 
respect to $\phi$. We can rephrase question (2) as: for which set systems $(E, \mathcal{A})$ and which functions $\phi$ is the corresponding kernel system a kernel matroid? Note that, generally, we shall take matroids to be defined in terms of their independent sets.

Example 2 The following array shows a small instance of the weighted bipartite matching problem, perhaps representing five conference delegates $V, W, X, Y$ and $Z$, expressing preferences for five study bedrooms $a, b, c, d$ and $e$ (blank entries indicate an unacceptable allocation):

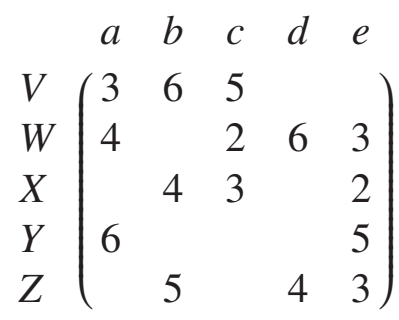

The set system in this case is $(E, \mathcal{A})$ where $E$ is the set of positive matrix entries and $\mathcal{A}$ is the set of perfect matchings of delegates to rooms. Let $\phi: \mathcal{A} \rightarrow \mathbb{R}^{\geq 0}$ map perfect matchings in $\mathcal{A}$ to their edge-weight sums. It can be calculated that $\mathcal{A}$ consists of ten perfect matchings, whose values form the set $\{18,20,22,24\}$. A maximum-weight matching is easily found by standard techniques but for the sake of example suppose we seek a kernel set for the system. We can find one of size 3: the entries $\{V c, W a, W e\}$ can be deleted from $E$ without changing the set of possible matching values. Although this is a reduction in the size of $E$ of only $15 \%$ the number of complete matchings is reduced by $60 \%$ to only four (one of each value, so the maximum possible reduction for a kernel set). This is the good news; the bad news is that the collection of all kernel sets does not, in this instance, form a matroid: $K_{1}=\{V c, W a\}$ and $K_{2}=\{V c, X c, Z e\}$ are both kernels but if any element of $K_{2}-K_{1}$ is added to $K_{1}$ the result is no longer a kernel, so the set system fails the augmentation axiom.

In section 5 we will see an optimisation problem where the kernel sets $d o$ form a matroid. Finding ourselves in the circumstances of Example 2 we could look for other ways to identify the maximum cardinality kernel sets; however, our interest in the rest of this paper lies in cataloguing cases where the kernel system is guaranteed to be a matroid, or is guaranteed not to be one. In fact, for the most part we shall begin with set systems which are already matroids-this is somewhat limiting from the optimisation point of view but seems theoretically to be a suitable starting point.

\section{Some basic examples}

We shall assume familiarity with the basic idea of a matroid. Our terminology will follow [7], with $M=(E, \mathcal{I})$ representing the matroid on ground set $E$ having independent set collection $\mathcal{I}$. Some constructions which we shall apply to $M=(E, \mathcal{I})$ are restriction, with $M \mid X, X \subseteq E$, being the matroid whose independent sets are precisely those of $M$ which are also subsets of $X$; and direct 
sum, with $M \oplus M^{\prime}, M^{\prime}=\left(E^{\prime}, \mathcal{I}^{\prime}\right), E \cap E^{\prime}=\emptyset$ being the matroid on $E \cup E^{\prime}$ whose independent sets are precisely all unions of members of $\mathcal{I}$ and $\mathcal{I}^{\prime}$. The notation $U_{k, n}$, for nonnegative integers $k \leq n$, denotes the $k$-uniform matroid in which the maximal independent sets are all $k$-subsets of $\{1, \ldots, n\}$.

Example 3 Let $E=\{a, b, c, d\}$ and let $\mathcal{A}=\{\emptyset, a, b, c, d, a b, a c, a d\}$, where for simplicity we are writing $a b$ for the set $\{a, b\}$, etc. Then $(E, \mathcal{A})$ is a matroid. Define the function $\phi$ by

$$
\phi(a)=\phi(b)=\phi(c)=1, \phi(d)=\phi(a c)=2, \text { and } \phi(\emptyset)=\phi(a b)=\phi(a d)=3 .
$$

Now $\operatorname{Ker}_{\phi}=\{\emptyset, a, b, c, d, a b, a c, b c, b d\}$ which is not a matroid since the exchange property fails for $b d$ and $a c$. Thus we see that a set $\operatorname{system}(E, \mathcal{A})$ certainly does not give a matroid for every choice of function $\phi$, even when the set system is itself a matroid. A small change can change things dramatically - if $\phi(a c)$ is instead defined to have value 3 , then the kernel system is now a matroid, being isomorphic to $U_{2,3} \oplus U_{0,1}$.

Example 4 Let $E=\{a, b, c, d\}$ and let $\mathcal{A}=\{\emptyset, a, b, c, d, a b, b c, c d, a d\}$. Again the system $M=$ $(E, \mathcal{A})$ is a matroid. Let $w_{1}$ and $w_{2}$ be weight functions defined on $E$ by

$$
\begin{gathered}
w_{1}(a)=w_{1}(b)=w_{1}(c)=1, w_{1}(d)=2, \text { and, } \\
w_{2}(a)=w_{2}(b)=1, w_{2}(c)=w_{2}(d)=2 .
\end{gathered}
$$

Define two functions on $\mathcal{A}$ by

$$
\phi_{i}(\emptyset)=0 \text { and } \phi_{i}(X)=\max _{x \in X} w_{i}(x), i=1,2 .
$$

We have

$$
\begin{aligned}
\operatorname{Ker}_{\phi_{1}} & =\{\emptyset, a, b, c, a b, a c, b c\} \\
\operatorname{Ker}_{\phi_{2}} & =\{\emptyset, a, b, c, d, a c, a d, b c, b d\}
\end{aligned}
$$

Now $\operatorname{Ker}_{\phi_{1}}$ and $\operatorname{Ker}_{\phi_{2}}$ are again both matroids; $\operatorname{Ker}_{\phi_{1}}=U_{2,3}$, the uniform matroid of rank 2 on three elements; $\operatorname{Ker}_{\phi_{2}}$, meanwhile, is isomorphic to the original matroid $M$.

In fact, the choice of functions $\phi_{i}$ in Example 4 makes the structure of $\mathcal{A}$ irrelevant: $K$ is a kernel set if and only if

$$
\left\{\phi_{i}(x) \mid x \in E-K\right\} \cup\left\{\phi_{i}(\emptyset)\right\}=\operatorname{Im}\left(\phi_{i}\right) .
$$

Defining $\phi$ to be the max or min functions is a special case of where supersets of the ground set inherit subsets of ground set $\phi$ values, so that they do nothing to help preserve the image of $\phi$.

A final example will touch on the idea of representability. 
Example 5 Let $\mathcal{A}$ be the collection of independent sets of the uniform matroid $U_{k, n}$, for some $k \leq n$. For $s \leq k \in \mathbb{Z}^{\geq 0}$, define $\phi_{s}$, for subsets $X$ in $\mathcal{A}$, by

$$
\phi_{s}(X)= \begin{cases}1 & \text { if }|X| \geq s \\ 0 & \text { otherwise. }\end{cases}
$$

Then $\operatorname{Ker}_{\phi_{s}}(\mathcal{A})$ is the uniform matroid $U_{n-s, n}$.

We may examine different values of $k$ and $s$ in Example 5. If $s=k$ then $\operatorname{Ker}_{\phi_{s}}(\mathcal{A})$ is the dual of $U_{k, n}$. If $k \geq n / 2$ and $s=n-k$ then $\operatorname{Ker}_{\phi_{s}}(\mathcal{A})$ is again $U_{k, n}$. Now consider the case $k=n-1, s=n-2$. Then $U_{k, n}=U_{n-1, n}$ is graphic $\left(G=C_{n}\right.$, the $n$-cycle), cographic ( $G$ consists of $n$ parallel edges) and binary (representable over $\mathrm{GF}(2)$ - consider the incidence matrix of $C_{n}$ ). On the other hand, when $n>3, \operatorname{Ker}_{\phi_{s}}(\mathcal{A})=U_{2, n}$ is none of these things. Thus Example 5 supplies matroids which are not graphic/cographic/ $F$-representable over a field $F$ but are given as kernel matroids of matroids which are graphic/cographic/ $F$-representable. This generalises easily as we will see in the next section.

\section{Some basic results}

Proposition 6 Let $M$ be a matroid of rank $r$ on ground set $E$. On the same ground set, let $\mathcal{A}$ be the collection of independent sets of the uniform matroid $U_{k, n}$, for some $k, n-r \leq k \leq n$. Define $\phi$, for subsets $X$ in $\mathcal{A}$, by

$$
\phi(X)= \begin{cases}1 & \text { if } E-X \text { is independent in } M \\ 0 & \text { otherwise }\end{cases}
$$

Then $\operatorname{Ker}_{\phi}(\mathcal{A})=M$.

Proof Let $X$ be a subset of $E$ and consider the effect on $\operatorname{Im}(\phi)$ of deleting $X$. We always retain zero in $\operatorname{Im}(\phi)$ since $E-\emptyset=E$ is never independent in $M$ unless $M=U_{n, n}$, in which case zero is not in $\operatorname{Im}(\phi)$. So it is enough to show that 1 is retained if and only if $X$ is independent in $M$.

Suppose $X$ is a base of $M$. Then $\phi(E-X)=1$ since $E-(E-X)=X$ which is independent. Moreover, $E-X$ is in the domain of $\phi$ because $|E-X|=n-r$ and $\phi$ is defined on all subsets of $E$ of size not exceeding $k \geq n-r$. So $\operatorname{Im}(\phi)$ retains 1 on deletion of $X$. By heredity, this extends to all independent sets of $M$.

If $X$ is not independent in $M$ then no set $Y \subseteq E-X$ can have $\phi(Y)=1$ because this would mean that $E-Y$ was independent in $M$. But this would make $X \subseteq(E-Y)$ independent by the hereditary property, which is a contradiction.

We can determine $\operatorname{Ker}_{\phi}(\mathcal{A})$ for some generic functions $\phi$ even when the collection $\mathcal{A}$ has very few restrictions placed on it: 
Proposition 7 Let $(E, \mathcal{A})$, be a set system and for some set $S$ let $\phi: \mathcal{A} \rightarrow S$ be a function. Let $n$ denote $|E|$. Then

1. if $\phi$ is injective, and every element of $E$ belongs to at least one set in $\mathcal{A}$, then $\operatorname{Ker}_{\phi}$ is isomorphic to the rank zero matroid $U_{0, n}$;

2. if $\phi$ is the constant function, $\phi(X)=s$ for all $X \in \mathcal{A}$, and $\mathcal{A}$ contains the empty set then $\operatorname{Ker}_{\phi}$ is isomorphic to the free matroid $U_{n, n}$;

3. if $(E, \mathcal{A})$ is a matroid, $M$, say (defined as usual in terms of its independent sets) and $\phi$ maps independent sets $X$ to their rank, which is to say their cardinality $|X|$, then $\operatorname{Ker}_{\phi}=M^{*}$, the dual of $M$.

\section{Proof}

1. Suppose $x \in E$. Then $x \in X$ for some $X \in \mathcal{A}$ and $\phi(X)$ is unique in the image of $\phi$. Therefore $x$ belongs to no set of $\operatorname{Ker}_{\phi}(\mathcal{A})$.

2. We may remove the whole set $E$ and, since $\phi(\emptyset)=s$, the image of $\phi$ is still preserved. Since $\operatorname{Ker}_{\phi}$ is subset-closed it must have all subsets of $E$. So $\operatorname{Ker}_{\phi}=U_{n, n}$.

3. Let $M$ have rank $r$, for some $r \leq n$. Then $\operatorname{Im}(\phi)=\{0,1, \ldots, r\}$. Let $B$ be a basis of $M$. We must show that $B^{\prime}=E-B$ is a maximal subset of $\operatorname{Ker}_{\phi}$. Now $B^{\prime} \in \operatorname{Ker}_{\phi}$ because $M \mid B=U_{r, r}$, whose image under $\phi$ is again $\{0,1, \ldots, r\}$. And $B^{\prime}$ is maximal because if $B^{\prime \prime}=B^{\prime} \cup\{x\}$ for $x \notin B^{\prime}$ then $\left|E-B^{\prime \prime}\right|<r$, so $\phi$ restricted to $E-B^{\prime}$ cannot have $r$ in its image.

Conversely, suppose that $X$ is a maximal set in $\operatorname{Ker}_{\phi}$. We must show that $X$ is a cobasis of $M$. Now $\phi$ restricted to $E-X$ has image $\{0,1, \ldots, r\}$, so $E-X$ contains a basis of $M$. Suppose that it is not itself a basis. Then there is some $y \in E$ with $E-X-y$ also containing a basis of $M$. But then $X \cup\{y\}$ is also in $\operatorname{Ker}_{\phi}()$ contradicting the maximality of $X$.

Remark 8 1. In Proposition 7(1) we need the condition on the elements of $E$. For example, suppose that $E=\{a, b\}$ and $\mathcal{A}=\{\emptyset,\{a\}\}$, with $\phi(\emptyset)=0$ and $\phi(\{a\})=1$. Then $\phi$ is an injective function onto $\{0,1\}$. But $\operatorname{Ker}_{\phi}(\mathcal{A})=(E,\{\emptyset,\{b\}\})$, which is isomorphic to $U_{0,0} \oplus U_{1,1}$.

2. Proposition $7(2)$ is false if $\emptyset \notin \mathcal{A}$. For example, take $E=\{a, b, c, d\}$, and the system $(E,\{a, b c, c d\})$. Then with $\phi$ the constant function the set $\{b, c, d\}$ is maximal in $\operatorname{Ker}_{\phi}$ and is larger than $\{a, b\}$ which is also maximal. So $\operatorname{Ker}_{\phi}$ is not a matroid in this case.

3. Proposition 7(3) shows that, in the case of a self-dual matroid, we have $\operatorname{Ker}_{\phi}\left(\operatorname{Ker}_{\phi}(\mathcal{A})\right)=\mathcal{A}$, for some choices of $\mathcal{A}$ and $\phi$. This is not always the case since, for instance, the constant map $\phi$ can act idempotently, as in Proposition 7(2).

4. It does not seem obvious that Proposition 7(3) should have a 'multidimensional' analog; however, such is the case as we prove in section 5. 
If $\phi$ is some particular function then we can still determine $\operatorname{Ker}_{\phi}(\mathcal{A})$ in some specific cases. The next result replaces the $\phi$ function from Example 5 with a matroid function (referred to as corank by some authors):

Proposition 9 Let $M$ be $U_{k, n}$, the k-uniform matroid on ground set $E$ with $|E|=n$. Let the function $\phi$ be defined on the independent sets of $M$ by $\phi(X)=\operatorname{rank}(E-X)$. Then

$$
\operatorname{Ker}_{\phi}(M)= \begin{cases}U_{n, n} & \text { if } k \leq n / 2 \\ U_{n-k, n}=M^{*} & \text { if } k>n / 2\end{cases}
$$

Proof. For any independent set $X \subseteq E, \phi(X)=\min (n-|X|, k)$, giving $\operatorname{Im}(\phi)=\{\min (k, n-k), \ldots, k\}$. If $k \leq n / 2$ then $\min (k, n-k)=k$, so that the image is the singleton $\{k\}$; since $\phi(\emptyset)=\operatorname{rank}(E)=k$ we see that we can remove all elements of $E$ without disturbing this image and $\operatorname{Ker}_{\phi}(M)=U_{n, n}$. If, on the other hand, $k>n / 2$, then $\min (k, n-k)<k$ and we will lose elements of the image as soon as $|X|$ exceeds $n / 2$.

Recall that if set $E$ is partitioned into $t$ sets $E_{1}, \ldots, E_{t}$ then the partition matroid is defined to have independent sets equal to the partial transversals of the partition sets. Such a matroid is graphic, being isomorphic to the cycle matroid $M(G)$ of the graph $G$ consisting of a central vertex $u$ connected to an independent set of vertices $u_{1}, \ldots, u_{t}$ by $\left|E_{1}\right|, \ldots,\left|E_{t}\right|$ multiple edges, respectively. Using the more generally accepted terminology, the corank of a subset $X$ of $E$ can then be specified as the size of a maximum non-cutset of $G$ chosen from $X$. Suppose we take $\mathcal{A}$ to be the collection of independent sets of $M(G)$, i.e. the forests in $G$; and consider the function $\phi: \mathcal{A} \rightarrow \mathbb{Z}^{\geq 0}$ defined to be the corank function; then we can ask what is $\operatorname{Ker}_{\phi}(M(G))$.

An example is shown in figure 1. The function $\phi$, defined to be the corank function, has image $\{0,1,2\}$ for the graph in (a). The subset of edges $\{b, e, g\}$ shown in (b) belongs to $\mathcal{A}$ and the restriction of $\phi$ to this set retains the whole image, because the coranks of $\{b, e, g\},\{e, g\}$ and $\{g\}$ are 2,1 and 0 , respectively. The edge subset $\{a, c, d, f\}$ is therefore a kernel set because those independent sets which involve none of these edges, i.e. forests in figure 1(b), still produce all the original corank values, with respect to the graph in figure 1(a).

It is easy to generalise from this example to the case of the corank function for a general partition matroid: the non-cutsets of $G$ consist of up to $\left|E_{i}\right|-1$ edges from each set of multiple edges in $G$ having multiplicity greater than 1 ; we may also remove all the edges of multiplicity 1 , since these edges contribute zero to the corank, as does the empty set. Thus we have:

Proposition 10 Suppose $E$ is a set and $M$ is the partition matroid on a partition $E_{1}, \ldots, E_{t}$ of $E$ into non-empty parts. Let $\phi$ be the corank function on $M$, let $n_{i}=\left|E_{i}\right|$, for $i=1, \ldots, t$ and suppose that there are s parts of size 1 in the partition. Then

$$
\operatorname{Ker}_{\phi}(M)=U_{s, s} \oplus\left(\bigoplus_{n_{i} \neq 1} U_{n_{i}-1, n_{i}}\right) .
$$




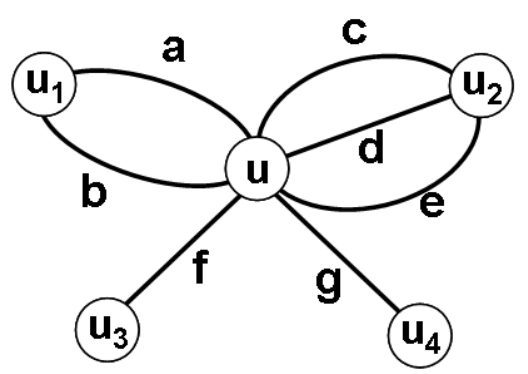

(a)

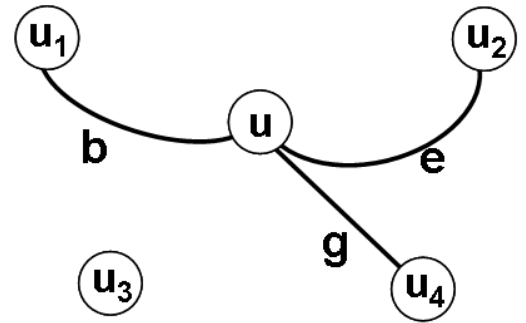

(b)

Figure 1: (a) graph whose cycle matroid is isomorphic to the partition matroid of $\{\{a, b\},\{c, d, e\},\{f\},\{g\}\}$ (b) a subset of edges with rank 3 and corank 2 .

\section{Sum-free sets-a negative result}

So far we have concentrated on looking for examples of functions on set systems which yield kernel matroids. Now we look at the other side of the coin and exhibit a case in which the kernel system is essentially never a matroid. Let $E$ be a set of positive integers and let $\mathcal{A}$ be the power set of $E$ (so that $(E, \mathcal{A})$ is the free matroid in which every subset is independent). Define $\phi$ to be the function on $\mathcal{A}$ which takes subsets to 1 if they are sum-free and 0 otherwise, where a set $X$ is sum-free if no sum of two elements in $X$ is again in $X$. For example, if $E=\{1,2,4,5\}$ then $\phi(\{1,2\})=\phi(\{1,3,4\})=0($ since $1+1=2$ and $1+3=4)$ while $\phi(\{2,3\})=\phi(\{1,3,5\})=1$. Sumfree sets have a rich literature centering around the recently-proved Cameron-Erdôs Conjecture $[6,8]$; some of their properties could surely be formulated in terms of $\operatorname{Ker}_{\phi}$ but our aim is merely to show that this kernel system cannot obey the augmentation axiom for matroids, except in a few trivial cases.

Recall that a bridge in a matroid is an element which belongs to no dependent set and will therefore appear in every basis. We will exclude such elements from our analysis since they are uninteresting. For example, the set $E=\{1,2,5,8,11,14,17\}$ contains just one summation: $1+1=2$. With the above definition of $\phi$, any subset $X$ of $E$ including $\{1,2\}$ will have $\phi(X)=0$ and this is the only way that 0 can appear in the image of $\phi$. So no kernel set with respect to $\phi$ can contain either of 1 or 2 but any maximal kernel set will contain all of 5,8,11,14 and 17: this arithmetic progression consists of bridges. We could extend this arithmetic progression to any arbitrary length; all such extensions will give a kernel matroid which, up to addition of bridges, is still just $U_{0,2}$

Theorem 11 Let $E, \mathcal{A}$ and $\phi$ be as above and suppose that $\operatorname{Ker}_{\phi}(\mathcal{A})$ is a bridgeless matroid. Then it is one of

$$
\begin{array}{rlll}
\text { (I) } & U_{0,2}, & \text { (II) } & U_{0,1} \oplus U_{1,2}, \\
\text { (III) } & U_{0,3}, & \text { (IV) } & U_{0,2} \oplus U_{1,2} .
\end{array}
$$

Proof. Suppose that $\operatorname{Ker}_{\phi}(\mathcal{A})$ is a bridgeless matroid: we will denote it by $M^{*}$ for notational 
convenience since we going to work with its dual; this dual matroid is now $M$.

Let $B$ be a base of $M^{*}$. Then $B$ is a maximal kernel set, so in particular it is a maximal set whose deletion leaves a subset $X$ of $E$ for which $\phi(X)=0$, i.e. $X$ must be precisely the components of a single summation, $X=\{x, 2 x\}$ or $X=\{x, y, x+y\}$. Now in the dual matroid $M$ these $X$ are the possible bases. Thus $M$ has rank 2 or 3 .

Suppose $\operatorname{rank}(M)=2$ and that $\{x, 2 x\}$ is a base of $M$, call it $B_{1}$. If the ground set $E$ has no other elements than $B_{1}$ then we have case (I). Now suppose that $y$ is an element of $E$ not in $B_{1}$. Since $y$ is not a bridge in $M^{*}$ it cannot be a loop in $M$ so it is independent. So by the augmentation axiom, we have a second base, $B_{2}$ say, which is either (a) $\{x, y\}$ implying $y$ is $x / 2$ (it cannot be $2 x$ since $y \notin B_{1}$ ); or (b) $\{2 x, y\}$, implying $y$ is $x$ (it cannot be $4 x$ since $y \notin B_{1}$ ). In each case, we see that the pair $\left(B_{1}, B_{2}\right)$, up to ordering, is of the form $(\{a, 2 a\},\{2 a, 4 a\})$ for some positive integer $a$. The same argument now shows that adding a third base will violate the exchange axiom. So $E=\{a, 2 a, 4 a\}$ and we have case (II).

Now suppose that $\operatorname{rank}(M)=3$, and that $M$ has a base $B=\{x, y, x+y\}$. If this is the only base then since $M^{*}$ is bridgeless and therefore $M$ contains no loops, we have $E=B$ and $M=U_{3,3}$. So $M^{*}=U_{0,3}$ which is case (III) (note that this excludes the case where $E$ has the form $(a, 2 a, 3 a)$ since the kernel matroid then has $3 a$ as a bridge).

Recall the exchange axiom: if we have two bases $B_{1}$ and $B_{2}$ then for each $e \in B_{1}-B_{2}$ there is an element $f \in B_{2}-B_{1}$ such that we have a third (not necessarily distinct) base $B_{1}-e+f$, call it $B_{3}$.

Note that each element in a sumset is either the sum or (the absolute value of) the difference of the other two elements. So if there is more than one base in $M$ and the exchange axiom is to be applied to $e \in B_{1}$ then, if $e$ is the sum (difference) of the other two elements in $B_{1}$, the difference (sum) of the other two elements in $B_{1}$ will certainly be in $B_{2}$. Thus $B_{3}$ can take one of two forms and in fact is equal to $B_{2}$.

Applying this to our base $B=\{x, y, x+y\}$, we see that a second base must be of the form $B^{\prime}=$ $\{y, x+y, x+2 y\}$ or $B^{\prime \prime}=\{|x-y|, x, y\}$. There cannot be a third base as $B^{\prime}$ and $B^{\prime \prime}$ are incompatible with respect to the exchange axiom and applying the above argument to either of them gives a triple that is incompatible with $B$.

We conclude that $E$ has the form $\{a, b, a+b, a+2 b\} ;\{a\}$ and $\{a+2 b\}$ are kernels but neither of the other elements can be deleted without removing both bases and therefore all elements for which $\phi$ has value 0 . So $M=U_{0,2} \oplus U_{1,2}$, which is case (IV).

\section{Kernels of matroids with coloured ground sets}

Our final result is another illustration of how kernel systems might play a role in optimisation, in that we derive a kernel matroid for a problem which cannot itself be solved greedily. 


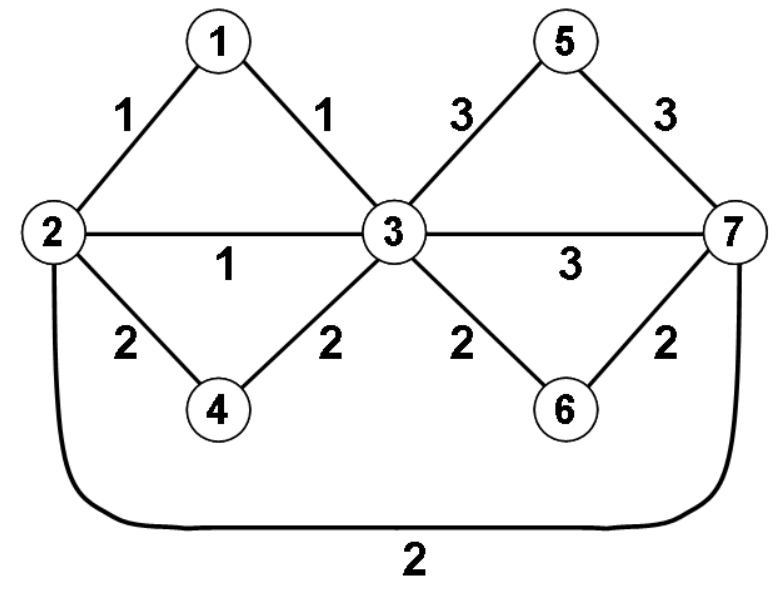

(a)

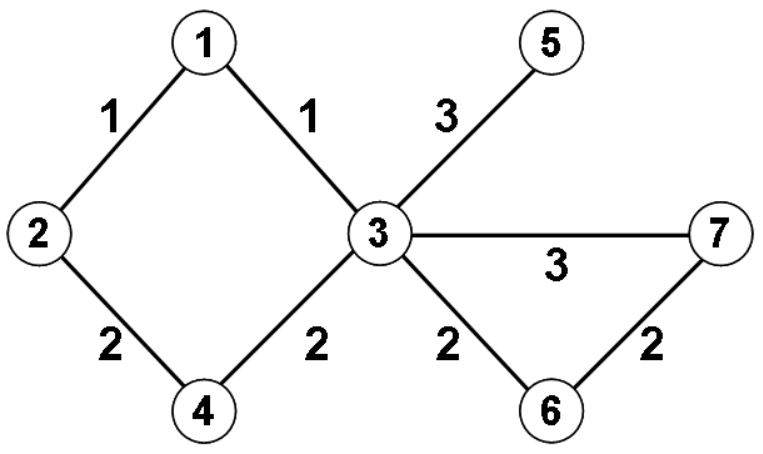

(b)

Figure 2: (a) graph with edges coloured from the colour set $\{1,2,3\}$ and (b) with a union of maximal non-cutsets removed

Theorem 12 Let $M=(E, \mathcal{A})$ be a matroid defined on $E$ and suppose that $E$ is partitioned into sets $E_{1}, \ldots, E_{t}$. Define a function $\phi: \mathcal{A} \rightarrow \mathbb{Z}^{t}$ by $\phi: X \mapsto\left(x_{1}, \ldots, x_{t}\right)$ with $x_{i}=\left|X \cap E_{i}\right|, i=1, \ldots, t$. Then $\operatorname{Ker}_{\phi}(M)=M_{1}^{*} \oplus \ldots \oplus M_{t}^{*}$ where $M_{i}=M \mid E_{i}$, the restriction of $M$ to $E_{i}$.

We remark that Proposition 7(3) is precisely the case $t=1$. Before we give the proof of this result it is worth illustrating it in a concrete case. Suppose $G$ is a graph whose edges are coloured with the integers $1, \ldots, t$. For each forest $F$ in $G$ record the number of edges of each colour class - this vector is the value of the function $\phi$ on $F$. The theorem says that the kernel sets are obtained by taking the subgraphs induced by each colour and taking a non-cutset in each subgraph: the union of such non-cutsets is a kernel set. For the graph in figure 2(a), the image of $\phi$ is the set of three vectors $\{(1,3,2),(2,2,2),(2,3,1)\}$, together with all vectors which are componentwise less than these; for example the spanning tree $\{12,24,27,36,35,37\}$ gives the vector $(1,3,2)$ while the forest $\{12,24,27,35\}$ gives the vector $(1,2,1)$.

In contrast to finding a minimum-weight spanning tree (Example 1 in section 1 ) our vector $\phi$ suggests optimisation problems which cannot be solved greedily. For example, suppose we wish to find a spanning tree whose $\phi$ vector has minimum first coordinate: we must find a tree with vector $(1,3,2)$. Now the edges $\{23,35,37\}$ are a forest with $\phi$ vector $(1,0,2)$ but this forest cannot be extended to a spanning tree with vector $(1,3,2)$. However, theorem 12 says that any choice of maximal non-cutsets from each of the colour subgraphs will combine to give a kernel set. Such a kernel set has been removed in figure 2(b): it may be confirmed that all vectors in the image of $\phi$ may still be achieved in the resulting graph. The graph of figure 2(b) has 12 spanning trees compared to 128 in the original graph: as with the matching example in section 1 (Example 2) we have considerably simplified the search space; but this time kernel sets form a matroid and can be 
found greedily, so for this optimisation problem our approach will scale up.

The proof of theorem 12 follows easily using the definition of a matroid in terms of dependence axioms (see [9, section 1.7, exercises 7], for example ${ }^{2}$ ):

Definition 13 For a finite set $E$, let $\sim$ be a relation on $E \times 2^{E}$ satisfying, for any nonempty subsets $S$ and $T$ of $E$, and e any element of $E$ :

D1: $s \sim S$ for all $s \in S$;

D2: if $e \sim S$ and, for some $s \in S, e \nsim S-s$ then $s \sim S-s+e$;

D3: if $e \sim S$ and, for all $f \in S, f \sim T$ then $e \sim T$

Then the collection of subsets of $E$ which contain a subset $X$ and an element $x$ satisfying $x \sim X$ form the dependent sets of a matroid.

Proof of theorem 12. We first observe that $\operatorname{Ker}_{\phi}(M) \subseteq M_{1}^{*} \oplus \ldots \oplus M_{t}^{*}$. For suppose that we choose some set $K$ which contains a circuit of some component of the direct sum; without loss of generality we may say that $K \cap E_{1}$ contains a circuit of $M_{1}^{*}$. Then $E_{1}-K \cap E_{1}=E_{1}-K$ cannot contain a base of $M_{1}$. So the restriction of $\phi$ to $E_{1}-K$ has no vector of the form $\left(\operatorname{rank}\left(M_{1}\right), \ldots\right)$ in its image. But such vectors appear in the image of $\phi$. So every set $K$ in $\operatorname{Ker}_{\phi}$ must contain only independent sets of $M_{i}^{*}$ for every $i, 1 \leq i \leq t$.

Now suppose that $K$ is an independent set of $M_{1}^{*} \oplus \ldots \oplus M_{t}^{*}$ and let the restriction of $\phi$ to $E-K$ be denoted by $\phi_{K}$; we must show that $\phi_{K}$ preserves all image points of $\phi$. Let $X$ be an independent set of $M$ with $\phi(X)=\left(x_{1}, \ldots, x_{t}\right)$. Suppose, without loss of generality, that $e \in X$ is contained in $K \cap E_{1}$, so that $\phi_{K}(X)=\left(x_{1}^{\prime}, \ldots\right)$ where $x_{1}^{\prime}<x_{1}$. We must show that there exists some $f$ in $E_{1}-K$ with $X-e+f$ an independent set. Suppose for a contradiction that no such $f$ exists. Now let $S=E_{1}-K$ and let $T=X-e$. Then for all $f \in S$ we have $f \sim T$. But then by D3 of definition 13 we have $e \sim T$ contradicting the independence of $X$. So $\phi_{K}$ has larger first coordinate on $T+f$ than on $X$ and thus we can recover all vectors in the image of $\phi$ inductively.

Given a graph $G$ with edges coloured with positive integers an arithmetical alternative would be to define $\phi$ on forests of $G$ as the sum of their edge colours. Thus the spanning trees in the graph of figure 2(a) would now take values in $\{11,12,13\}$. We might hope to still get a kernel matroid for this version of $\phi$ but this is not the case: for example, either of the edge subsets $\{23,27,37\}$ or $\{13,34,37,67\}$ can be removed without changing the set of possible spanning tree values but this pair of sets does not satisfy the augmentation axiom, so there is no possibility of getting a matroid.

\footnotetext{
${ }^{2}$ Welsh mentions that these dependence axioms constitute the formulation of matroid theory by van der Waerden in 1937.
} 


\section{Concluding remarks}

The idea of a kernel system viewed, say, as a question about functions on hypergraphs, is extremely general. Nevertheless it seems that in specific instances it can provide a different way of looking at subset families and can yield some simple and intuitive results. It seems worthwhile, at any rate, continuing to catalogue the kinds of matroids that can arise and the kinds of optimisation problems that might be tackled by this route as a result.

An obvious question in connection with our approach is: why remove maximum cardinality subsets of the domain under preservation of the image-why not look for minimum cardinality subsets which will achieve the image? In the case where $\operatorname{Ker}_{\phi}(\mathcal{A})$ is a matroid this dual problem will clearly give the dual matroid and it may be that this dual matroid is a more natural setting for the problem. In this case the utility of the kernel system approach might be just that: an optimisation problem such as one of those given in section 1 may be reformulated in a different guise (this is, of course, a standard approach to problem solving).

As well as a dual problem, we have an inverse problem: given a set system $(E, \mathcal{A})$, when is it a kernel system? More precisely, when is there a second set system $\left(E, \mathcal{A}^{\prime}\right)$ and a function $\phi: \mathcal{A}^{\prime} \rightarrow S$ for which $\operatorname{Ker}_{\phi}\left(\left(E, \mathcal{A}^{\prime}\right)\right)=(E, \mathcal{A})$ ? If such a $\mathcal{A}^{\prime}$ and $\phi$ can be found, what can we say about them? An example of this problem was given at the end of section 2.

We will end with some questions which seem to us to point to areas which might fruitfully be explored.

Turán-type problems The classical Turán problem asks, given a fixed graph $G$, how many edges can an $n$-vertex graph have without containing $G$ as a subgraph. If we invert this, we can ask how many edges may be deleted from $K_{n}$ without removing the possibility of $G$ being a subgraph. Let $\mathcal{A}$ be the set of edge subsets of $K_{n}$ and define $\phi: \rightarrow\{0,1\}$ by $\phi(X)=1$ if $X$ contains a copy of $G$ and $\phi(X)=0$ otherwise. Now we can ask about the structure of $\operatorname{Ker}_{\phi}(\mathcal{A})$. For example, if $n=4$ and $G$ is a path consisting of two edges then it is not hard to see that $\operatorname{Ker}_{\phi}$ is the rank 4 matroid isomorphic to the cycle matroid of $K_{2,3}$. This is an illustration of the point we made in the introduction: there is no difference here between asking what subsets of edges we can remove while still retaining all $\phi$ values and asking what subsets of edges we should retain in order to achieve all $\phi$ values; in the above example the two questions are dual in the sense that they give rise to dual matroids. The idea of inverting Turán-type extremal problems goes back at least to the 70s (see [1], for example) but we are not aware of work on what structure the extremal or sub-extremal sets may have.

Universal graphs Suppose we extend the Turán problem to ask, given a set $\Gamma$ of graphs, how many edges may we delete from $K_{n}$ and still have every member of $\Gamma$ as a subgraph. Let $\mathcal{G}$ be the set of all unlabelled graphs and define $\phi: \mathcal{A} \rightarrow \mathcal{G}$ by $\phi(X)=$ the graph in $\mathcal{G}$ isomorphic to the subgraph defined by $X$. Again we can ask about $\operatorname{Ker}_{\phi}(\mathcal{A})$. In particular, if $\Gamma$ is the set of all $n$-vertex unlabelled trees, then we can ask what subsets of edges from $K_{n}$ can be removed and still allow every unlabelled $n$-vertex tree to be embedded. The maximum size of such 
an edge set was settled asymptotically by Chung and Graham in 1979 [1] (the proof is given in [2]) but again we can find no mention of the structural issue. Some related problems are given in [3] (section 3.5.1). We observe that the subsets of the edges of $K_{5}$ whose removal preserves all trees do not constitute the independent sets of a matroid but that all such subsets that are maximal have the same cardinality of four.

Duality If we have $\mathcal{A}$ and $\phi$ giving a kernel matroid $\operatorname{Ker}_{\phi}(\mathcal{A})=M$ then we can clearly take $M^{*}$, the dual of $M$. Now $M^{*}$ will again be a kernel matroid for certain subset families $\mathcal{A}^{\prime}$ and mappings $\phi^{\prime}$ (this is guaranteed by proposition 7(3)). We would like a concept of formal duality in which $\mathcal{A}^{\prime}$ can be chosen as a formal dual of $\mathcal{A}$ and similarly $\phi^{\prime}$ of $\phi$. In the case where $\mathcal{A}$ is itself a matroid then proposition 7(3) gives a solution when $\phi$ is the cardinality (or rank) function, so it would be desirable that our concept of formal duality is consistent with this.

Representability Given a matroid which is not representable over a given field, or is not algebraic, when can we construct it as a kernel matroid of a representable/algebraic matroid? Example 5 in section 2 gave examples for GF(2) and Proposition 6 extended this to any non-representable matroid. However the construction was limited to $0-1$ functions $\phi$; it would be of interest to find constructions using functions, which reflect more of the matroid structure.

Construction of classes of matroid We saw, in particular, in Example 5, that $U_{2,4}$, the excluded minor for $\mathrm{GF}(2)$-representability, could be given as a kernel matroid of a binary matroid. This suggests a question along the lines of: if a class $\mathcal{M}$ of matroids is characterised by excluded minors and these minors can be given as kernel matroids of matroids in $\mathcal{M}$, then can any non- $\mathcal{M}$ matroid be given as a kernel matroid of a matroid in $\mathcal{M}$ ? $U_{2,4}$ is an excluded minor for graphic and cographic matroids; so a concrete problem in the right direction would be: construct the other 4 excluded minors for these classes (see [7, Theorem 6.6.5]). A natural requirement would be that we use the same $\phi$ function in each case. There is, of course, a large body of theory to which this question is closely related (see [5], for example).

Identity Given a set family $\mathcal{A}$, does there exist a function $\phi$ for which $\operatorname{Ker}_{\phi}(\mathcal{A})$ is isomorphic to $\mathcal{A}$ ? Again proposition 7(3) gives a positive answer in the case where $\mathcal{A}$ is a self-dual matroid. Another instance was seen in Example 4 where the system $\operatorname{Ker}_{\phi_{2}}(\mathcal{A})$ was isomorphic to $\mathcal{A}$. If $\mathcal{A}$ is not a matroid then notice that it must still be subset-closed, since $\operatorname{Ker}_{\phi}$ will be.

Inverse problem This is another problem mentioned above: given two families on ground set $E$, say, $\left(E, \mathcal{A}_{1}\right)$ and $\left(E, \mathcal{A}_{2}\right)$ find a function $\phi$ such that $\mathcal{A}_{2}=\operatorname{Ker}_{\phi}\left(\mathcal{A}_{1}\right)$.

Acknowledgements The generalisation of Example 5 to give Proposition 6 was suggested to us by Tony Forbes. We are pleased to acknowledge helpful discussions with Bill Jackson and Taoyang Wu. The work of Chung and Graham on universal graphs was brought to our attention by Emil Vaughan and by Francesca Merola. An anonymous referee corrected a good many errors and made some helpful suggestions on presentation. 


\section{References}

[1] Chung, F.R.K. and Graham, R.L., "On universal graphs", Annals of the New York Academy of Sciences, 319, 1979, 136-140.

[2] Chung, F.R.K. and Graham, R.L, “On universal graphs for spanning trees”, J. London Math. Soc. 27 (2), 1983, 203-211.

[3] Chung, F.R.K. and Graham, R.L, Erdôs on Graphs: His Legacy of Unsolved Problems, AK Peters, 1998.

[4] Frank, A., "Kernel systems of directed graphs", Acta Sci. Math., 41, 1979, 63-76.

[5] Geelen, J., Gerards, A.M.H. and Whittle, G., "Towards a matroid-minor structure theory", in Grimmett, G. et al (eds.), Combinatorics, Complexity, and Chance: A Tribute to Dominic Welsh, Oxford Lecture Series in Mathematics and its Applications, 34, Oxford University Press, 2007, 72-82.

[6] Green, B., “The Cameron-Erdős conjecture”, Bulletin of the London Mathematical Society 36, 2004, 769-778.

[7] Oxley, J.G., Matroid Theory, Oxford University Press, 1992.

[8] Sapozhenko, A.A. “The Cameron-Erdős conjecture”, Dokl. Akad. Nauk 393 (6) 2003, 749752.

[9] Welsh, D.J.A., Matroid Theory, Oxford University Press, 1976. 